\title{
Thymic stromal lymphopoietin (TSLP) secretion from human nasal epithelium is a function of TSLP genotype
}

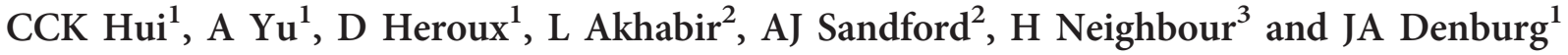

Recent candidate gene and genome-wide association studies have identified "protective" associations between the single-nucleotide polymorphism (SNP) rs1837253 in the TSLP gene and risk for allergy, asthma, and airway hyperresponsiveness. The absence of linkage disequilibrium of rs 1837253 with other SNPs in the region suggests it is likely a causal polymorphism for these associations, having functional consequences. We hypothesized that rs 1837253 genotype would influence TSLP secretion from mucosal surfaces. We therefore evaluated the secretion of TSLP protein from primary nasal epithelial cells (NECs) of atopic and nonatopic individuals and its association with rs1837253 genotype. We found that although atopic sensitization does not affect the secretion of TSLP from NECs, there was decreased TSLP secretion in NECs obtained from heterozygous (CT; 1.8-fold) and homozygous minor allele (TT; 2.5-fold) individuals, as compared with NECs from homozygous major allele individuals (CC; $P<0.05$ ), after double-stranded RNA (dsRNA) stimulation $\left(50 \mu \mathrm{g} \mathrm{ml}^{-1}\right)$. Our novel results show that $\mathrm{rs} 1837253$ polymorphism may be directly involved in the regulation of TSLP secretion. This may help explain the protective association of this genetic variant with asthma and related traits. Identifying functional consequences of SNPs in genes with previously reported clinical associations is critical in understanding and targeting allergic inflammation.

\section{INTRODUCTION}

Allergic diseases are often triggered by environmental stimuli that induce T helper type 2 cell (Th2) immune responses. For many years, the airway epithelium was recognized purely for its function as a physical barrier; however, it is now seen as an important initiator of the allergic response by secreting cytokines/chemokines that regulate innate immune cells. ${ }^{1,2}$ An epithelial cell-derived cytokine, thymic stromal lymphopoietin (TSLP), is interleukin-7-like, ${ }^{3}$ appears to be a key initiator of allergic inflammation, and also plays a role in the pathogenesis of allergic diseases-with effects on dendritic cells, ${ }^{4}$ T lymphocytes, ${ }^{5}$ and other effector cells such as mature eosinophils ${ }^{6,7}$ and basophils ${ }^{8}$ as well as their progenitors. ${ }^{9,10}$ In asthmatics, TSLP is expressed at elevated levels in the lungs and is linked to disease severity. ${ }^{11}$ Studies in murine models have demonstrated reduced airway disease in TSLP receptordeficient mice, whereas experimental lung-specific expression of a TSLP transgene induced experimental asthma (Th2 cytokine-associated inflammation of the airways). ${ }^{12}$ In a recent clinical trial, treatment with a human anti-TSLP antibody (AMG 157) reduced allergen-induced bronchoconstriction and airway inflammation in mild atopic asthmatics. ${ }^{13}$

Genome-wide association studies have shown associations between genetic variants in TSLP and allergic disease and phenotypes, including asthma ${ }^{14-16}$ and eosinophilia. ${ }^{17}$ Furthermore, a single-nucleotide polymorphism (SNP) rs1837253 in the TSLP gene has been shown to be associated with asthma, atopic asthma, and airway hyperresponsiveness. ${ }^{14-16,18-19}$ It has further been reported that the minor T allele of rs 1837253 in TSLP is associated with reduced risk of allergic rhinitis and asthma in males. ${ }^{20,21}$ The absence of linkage disequilibrium between rs1837253 and other SNPs, 2 Mb up- or downstream in the chromosomal region, suggests that it is likely causal, having functional consequences. ${ }^{18}$

\footnotetext{
${ }^{1}$ Department of Medicine, Division of Clinical Immunology and Allergy, McMaster University, Hamilton, Ontario, Canada. ${ }^{2}$ Centre for Heart Lung Innovation, University of British Columbia, St Paul's Hospital, Vancouver, British Columbia, Canada and ${ }^{3}$ Firestone Institute for Respiratory Health, McMaster University, Hamilton, Ontario, Canada. Correspondence: JA Denburg (denburg@mcmaster.ca)
} 
Recent evidence suggests that SNPs in the TSLP pathway may play a critical role in the pathogenesis of allergic disease, but the functional role of rs1837253 SNP in TSLP has not been previously described. Given that the T allele of the rs1837253 SNP in TSLP is associated with reduced risk of allergic disease, and as there are no other SNPs in linkage disequilibrium with rs1837253, we hypothesized that there would be associations between rs1837253 genotype and ex vivo production of TSLP, a crucial cytokine for the induction of Th2 inflammatory responses. Specifically, we hypothesized that individuals with the minor allele would have a decreased propensity to induce a Th2 inflammatory response related to an altered mediator profile, leading to "protection" from asthma and related traits. We therefore evaluated the double-stranded RNA (dsRNA)induced secretion of TSLP from primary nasal epithelial cells (NECs) from nonatopic and atopic individuals, examining associations among rs1837253 genotype, atopy, and TSLP secretion.

\section{RESULTS}

\section{Subject characteristics}

From 30 October 2013 to 10 February 2014, 61 subjects were recruited. On visit 1 , all 61 subjects received skin prick tests and had mouthwash samples collected and genotyped for the SNP rs1837253: 29 were homozygous for the major allele (CC), 9 were homozygous for the minor allele (TT), and 23 were heterozygous (CT). On visit 2, only 43 of these subjects returned and received nasal scrapes. Of these latter 43 subjects, 36 longterm expansions of NECs in vitro were successful. Of these 36 subjects, the breakdown by genotype was as follows: 14 CC, 8 TT, and 14 CT. Of the 18 subjects who dropped out before visit 2, 14 were because of studying/exam and holiday schedule conflicts and 4 were because of a common cold. Further subject characteristics are shown in Table 1.

\section{Polyl:C induces TSLP expression in NEC}

Polyinosinic:polycytidylic acid (polyI:C) has been previously reported to induce TSLP production (messenger RNA (mRNA) or protein) in human airway epithelial cells. ${ }^{22-24}$ To investigate

Table 1 Subject characteristics

\begin{tabular}{lccc}
\hline Characteristics & $\begin{array}{c}\text { Homozygous } \\
\text { major allele (CC) }\end{array}$ & $\begin{array}{c}\text { Heterozygous } \\
\text { (CT) }\end{array}$ & $\begin{array}{c}\text { Homozygous } \\
\text { minor allele (TT) }\end{array}$ \\
\hline Sample size, $n$ & 14 & 14 & 8 \\
$\begin{array}{l}\text { Sex, } \\
\text { male:female }\end{array}$ & $5: 9$ & $11: 3$ & $3: 5$ \\
Age, ${ }^{\text {a }}$ years & $28.4(15.0)$ & $30.4(15.4)$ & $27.0(11.0)$ \\
Atopy, $n$ & 6 & 4 & 4 \\
& & & \\
Ethnicity & $3(21.4)$ & $4(28.6)$ & $6(75.0)$ \\
Asian & $10(71.4)$ & $8(57.1)$ & $2(25.0)$ \\
Caucasian & $1(7.2)$ & $2(14.3)$ & $0(0.0)$ \\
Other & & &
\end{tabular}

Values in parentheses indicate percentages.

aMean (s.d.). whether NECs behave similarly, the epithelial nature of the cultured cells was confirmed using immunofluorescence staining for cytokeratin-5 (Figure 1), and cultured cells were stimulated with polyI:C for $0,3,6$, and $24 \mathrm{~h}$. PolyI:C $\left(25 \mu \mathrm{g} \mathrm{ml}^{-1}\right)$ induced a time-dependent expression of TSLP mRNA $(P<0.001$ at $6 \mathrm{~h}$; Figure 2a). Furthermore, a dosedependent release of TSLP protein was observed following $24 \mathrm{~h}$ stimulation with polyI:C $(P<0.01$; Figure 2 b) .

\section{Effects of atopy and TSLP rs1837253 genotype on TSLP expression}

No statistically significant association was found between allergic sensitization and production of TSLP by NECs at baseline. Furthermore, polyI:C did not induce differential TSLP secretion from NECs derived from nonatopic, as compared with atopic, individuals (Figure 3). However, polyI:C did induce differential TSLP secretion according to genotype (Figure 4a). When subjects were stratified by genotype, decreased TSLP secretion was observed in NECs obtained from heterozygous (CT; 1.8 -fold; $P<0.05)$ and homozygous minor allele (TT; 2.5 -fold; $P<0.05$ ) individuals, as compared with NECs from homozygous major allele (CC) individuals, after stimulation with polyI:C (25 and $50 \mu \mathrm{g} \mathrm{ml}^{-1}$; Figure $4 b, c)$.

\section{DISCUSSION}

There is increasing evidence implicating TSLP polymorphisms in the development of allergy and asthma. ${ }^{25-27}$ Previous work has documented inverse associations between the $\mathrm{T}$ allele of rs1837253 and asthma; ${ }^{18,20}$ however, the reasons for this association have remained unclear. Genetic polymorphisms are proposed to exert effects that can be measured through a variety of "intermediate" steps and outcomes ("phenotypes" that could be biological or clinical); however, information on these "intermediate phenotypes" is currently lacking.

In this study, our aim was to examine a key intermediate phenotype-specifically, TSLP secretion from NECs in response to stimulation ex vivo-that could shed insight into the underlying involvement of rs1837253 in the pathogenesis of

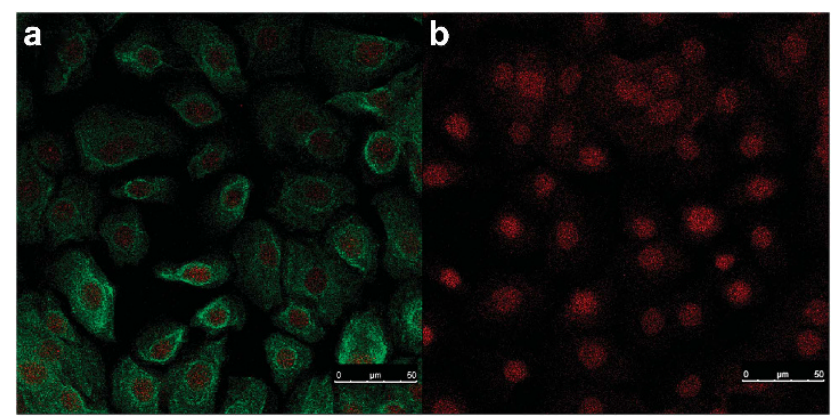

Figure 1 Immunofluorescence staining of cultured primary nasal epithelial cells (NECs). (a) Representative image of primary NECs stained for cytokeratin (green staining with AlexaFluor 488) to assess for the epithelial nature of the cells. (b) Representative image of negative control of primary NECs stained without primary antibody. Nuclei in all images stained red with DRAQ5 (original magnification $\times 63$ ) 
allergic disease. We selected the TSLP SNP rs1837253, based on the fact that it was the most significant signal in a candidate gene association study on asthma and related phenotypes, ${ }^{18}$ which has been confirmed in recent global genome-wide association study analyses. ${ }^{14-16}$ Although the role of TSLP in allergic rhinitis is definitely not as strong as the one it has been shown to play in asthma, we elected to use NECs in this study because of the ease in which NECs can be obtained compared with bronchial epithelial cells (BECs). Furthermore, although Zhang et al. $^{28}$ failed to find an association between specific SNPs in the TSLP gene and allergic rhinitis susceptibility in the Chinese population, Bunyavanich et al. ${ }^{21}$ reported the TSLP SNP rs1837253 to have reduced associations with allergic rhinitis in three independent cohorts for asthma. There is also accumulating evidence that suggests elevated levels of TSLP in nasal lavage ${ }^{29}$ higher levels of TSLP expression, ${ }^{30,31}$ and greater number of TSLP + cells $^{32}$ in the nasal mucosa of subjects with allergic rhinitis compared with healthy controls. Admittedly, a study with asthmatic subjects would more clearly elucidate the
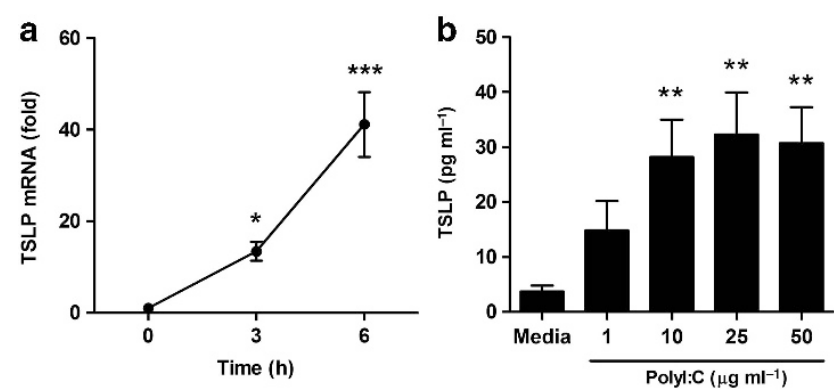

Figure 2 Polyinosinic:polycytidylic acid (polyl:C) induces thymic stromal lymphopoietin (TSLP) expression and secretion in human primary nasal epithelial cells (NECs). (a) Kinetics of TSLP expression in polyl:C $\left(25 \mu \mathrm{g} \mathrm{ml}^{-1}\right)$-stimulated NECs $(n=8)$. Results shown are expressed as fold change in the TSLP mRNA level in polyl:C-stimulated NECs at 3 and $6 \mathrm{~h}$ relative to the level in unstimulated NECs $(0 \mathrm{~h})$. (b) Primary NECs were incubated for $24 \mathrm{~h}$ with increasing concentrations of polyl:C $\left(0-50 \mu \mathrm{g} \mathrm{ml}^{-1}\right)$. Concentrations of TSLP protein in the culture supernatant were measured using enzyme-linked immunosorbent assay (ELISA). Results shown are mean \pm s.e.m. of duplicates $(n=8)$. One independent experiment performed per subject. Significant difference from unstimulated cells $\left({ }^{\star} P<0.05 ;{ }^{\star \star} P<0.01 ;{ }^{\star \star \star} P<0.001\right)$. functional significance of the TSLP SNP rs1837253; however, this was beyond the scope of this study.

Consistent with previous studies on small airway epithelial cells, ${ }^{22}$ NECs, ${ }^{33}$ and BECs from healthy and asthmatic individuals, ${ }^{23,34,35}$ we demonstrated that exposure to dsRNA in vitro induced a time-dependent expression of TSLP mRNA and a dose-dependent release of TSLP protein in NECs. $\mathrm{We}^{35}$ and others ${ }^{34,36,37}$ have previously reported differences in immune response between the BECs of healthy and asthmatic individuals, with enhanced TSLP secretion in BECs from asthmatics compared with their healthy counterparts following "viral" exposure. The higher capacity of asthmatic BECs to sense and respond to viral infections and thus to release elevated levels of TSLP was reported to be due, in part, to increased expression of the retinoic acid-inducible gene 1 (RIG1) in asthmatic airway epithelial cells following viral infections. ${ }^{36}$ In this study, dsRNA did not induce differential TSLP secretion in NECs obtained from nonatopic compared with atopic individuals, suggesting that there is no discrepancy in RIG-1 expression in NECs between these two groups. Further study examining differential RIG-1 expression in relation to clinical phenotypes will provide additional mechanistic insights into the function of SNP rs1837253.

Previous data demonstrate that the long form of TSLP is highly inducible by polyI:C in BECs. ${ }^{26} \mathrm{~A}$ functional SNP, rs3806933, has been identified in the regulatory region of the TSLP gene that enhances the binding of activator protein-1, a prominent transcription factor in airway diseases that regulates the expression of multiple inflammatory proteins. ${ }^{38}$ Furthermore, the rs3806933 SNP influences activator protein-1-driven promoter activity of the long form of TSLP in BECs. ${ }^{26}$ Activator protein-1 expression is enhanced in the asthmatic airway, ${ }^{38}$ consistent with reports that SNP rs3806933 is associated with adult asthma and childhood atopic asthma. ${ }^{27}$

In this study, we demonstrate that clinical atopic status does not affect NEC-derived TSLP. We however did not examine whether clinical atopic status was associated with rs1837253, as we would need a very large sample size in order to reach such a conclusion. Although it would be interesting to know other clinical details of the participants so that we could examine for
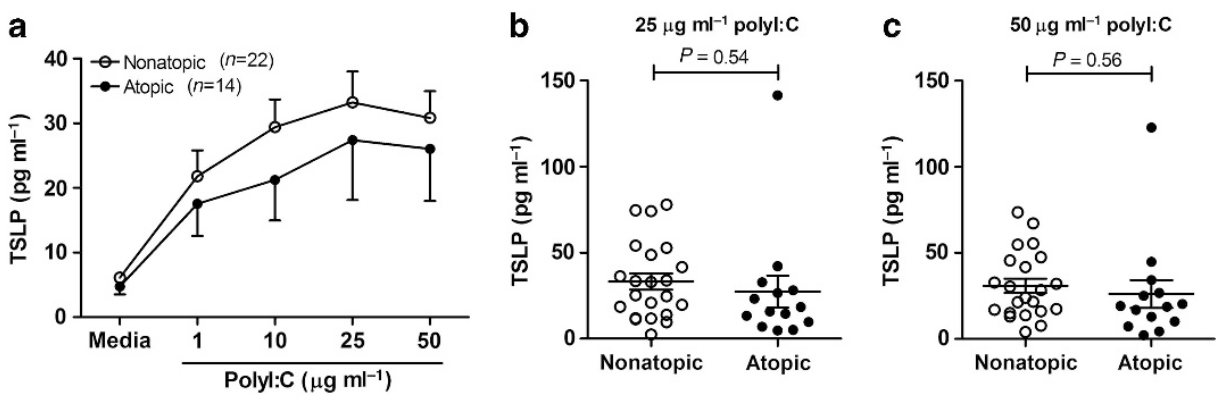

Figure 3 Relationship between atopy and polyinosinic:polycytidylic acid (polyl:C)-induced thymic stromal lymphopoietin (TSLP) secretion in nasal epithelial cells (NECs). Concentrations of TSLP protein in NEC culture supernatant from nonatopic $(n=22)$ and atopic $(n=14)$ individuals were measured using enzyme-linked immunosorbent assay (ELISA) following overnight stimulation with (a) increasing concentrations of polyl:C $\left(0-50 \mu \mathrm{g} \mathrm{ml}^{-1}\right)$; (b) $25 \mu \mathrm{g} \mathrm{ml}^{-1}$ of polyl:C; and (c) $50 \mu \mathrm{g} \mathrm{ml}^{-1}$ of polyl:C. Results shown are mean \pm s.e.m. of duplicates. One independent experiment performed per subject. 

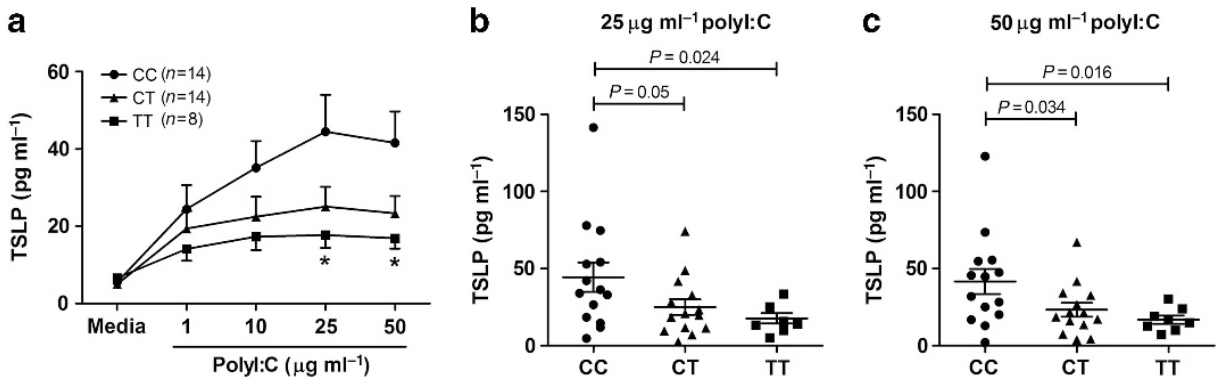

Figure 4 Relationship between TSLP rs1837253 genotype and polyinosinic:polycytidylic acid (polyl:C)-induced thymic stromal lymphopoietin (TSLP) secretion in nasal epithelial cells. Concentrations of TSLP protein in nasal epithelial cell culture supernatant from rs1837253 homozygous major allele (CC; $n=14)$, heterozygous (CT; $n=14)$, and homozygous minor allele (TT; $n=8$ ) individuals were measured using enzyme-linked immunosorbent assay (ELISA) following overnight stimulation with (a) increasing concentrations of polyl:C $\left(0-50 \mu \mathrm{g} \mathrm{ml}^{-1}\right)$; (b) $25 \mu \mathrm{g} \mathrm{ml}^{-1}$ of polyl:C; and (c) $50 \mu \mathrm{g} \mathrm{ml} \mathrm{m}^{-1}$ of polyl:C. Results shown are mean \pm s.e.m. of duplicates. One independent experiment performed per subject. Significant difference from CC genotype $\left({ }^{\star} P<0.05\right)$.

correlations between the clinical characteristics and TSLP production ex vivo, and/or TSLP genotype, unfortunately, aside from clinical atopic status, we do not have these. However, we $\mathrm{w}^{35}$ and others ${ }^{34,36,37}$ have previously demonstrated that primary BECs from patients with asthma release more TSLP than primary BECs from healthy controls after dsRNA stimulation. Furthermore, Harada et al. ${ }^{26}$ showed that individuals with the rs3806933 SNP in the promoter region of long-form TSLP have increased TSLP expression and protein secretion. Nonetheless, in this study, we related the differential secretion of TSLP protein to the SNP rs1837253 genotype, and our data suggest that this SNP in the upstream region of TSLP has functional effects on TSLP protein production. SNP rs 1837253 is located $5.7 \mathrm{~kb}$ upstream of the TSLP transcription start site and is predicted to disrupt a number of potential transcription factor binding sites. ${ }^{39}$ It is possible the rs1837253 $\mathrm{T}$ allele downregulates microbe-induced production of TSLP in NECs through inhibiting the binding of transcription factors to regulatory elements. In order to better understand the mechanism of SNP rs1837253 associations with TSLP secretion, potential differential binding of possible regulatory proteins to this important SNP should be examined. As such, rs1837253 SNP-associated epigenetic alterations, such as DNA methylation in airway epithelial cells, merits investigation.

In a recent proof-of-concept study, ${ }^{13}$ in addition to reducing allergen-induced bronchoconstriction in both early and late asthmatic responses, AMG 157, a human anti-TSLP monoclonal antibody, was demonstrated to reduce markers of systemic and airway inflammation measured as fraction of exhaled nitric oxide and sputum eosinophil levels, as well as levels of circulating eosinophils. The capacity for AMG 157 to decrease baseline blood eosinophil counts and exhaled nitric oxide $^{13}$ suggests a pivotal role for TSLP in the development or persistence of asthma in the absence of allergen exposure. In vivo, TSLP levels are elevated in asthmatics and are associated with disease severity and eosinophilic inflammation. ${ }^{40}$ Relevantly, we recently demonstrated the ability of TSLP to mediate eosinophil-basophil differentiation from human peripheral blood hemopoietic progenitor cells (hemopoiesis) ex vivo in relation to clinical atopic status, a process that was dependent on tumor necrosis factor- $\alpha$ and TSLP-TSLP receptor interactions. ${ }^{10}$ In this study, we demonstrate for the first time diminished TSLP secretion in NECs derived from individuals who are carriers of the rs1837253 $\mathrm{T}$ allele, a finding that provides key insight into allergic disease pathogenesis and explains, at least in part, the inverse association between rs1837253 and asthma and related phenotypes, and the recently reported therapeutic effects of TSLP antagonism. However, to what extent a reduction in TSLP levels is functionally and/or clinically significant in vivo in humans in the context of allergic disease is unknown. Nonetheless, several clinical outcomes and phenotypes of allergic diseases such as allergic rhinitis, total immunoglobulin E, and airway hyperresponsiveness have also been reported to be associated with SNPs in TSLP. ${ }^{18,20,21,25-27,41}$ Collectively, these data suggest a critical role for TSLP in regulating multiple downstream effector pathways involved in allergic disease.

Finally, asthma exacerbations are a major cause of mortality, and repeated exacerbations can cause permanent impairment of lung function. ${ }^{42}$ Rhinoviruses, single-stranded RNA viruses that synthesize dsRNA during replication at the respiratory epithelium, ${ }^{43}$ are a major cause of asthma exacerbations in both children and adults. ${ }^{44}$ PolyI:C is a highly accepted dsRNA surrogate commonly used to simulate viral infection, as it mimics many aspects of rhinovirus infection in primary BECs, ${ }^{34}$ allowing for the yield of robust and reproducible data. However, other strains of viruses, rhinovirus ${ }^{23}$ and respiratory syncytial virus, ${ }^{36}$ have demonstrated similar responses by inducing TSLP from airway epithelial cells. As such, our findings may have implications in explaining virus-induced asthmatic exacerbations, especially those because of rhinovirus infections. Of note, TSLP has recently been demonstrated to be a methylation-sensitive gene. ${ }^{45,46}$ Along these lines, our findings demonstrate differential secretion of TSLP between rs1837253 genotypes after polyI:C stimulation, suggesting that epithelial cells may be susceptible to epigenetic alterations induced by environmental factors such as infection. These epigenetic changes may, in turn, alter the threshold for epithelial activation in response to environmental stimuli such as viruses and allergens. ${ }^{47}$ For example, Toll-like receptor 
ligands have been demonstrated to alter methylation processes. ${ }^{48}$ Given that reduction of DNA methylation facilitates transcription by permitting transcription factors or coactivators to bind to regulatory elements (promoter or enhancer regions), ${ }^{49,50}$ we hypothesized that there would be enhanced methylation of the TSLP promoter in NEC obtained from heterozygous (CT) and homozygous minor allele (TT) individuals, following stimulation with polyI:C. ${ }^{45,46}$ Therefore, the effects of dsRNA and/or Toll-like receptor-mediated production of TSLP in NECs from patients with or without asthma in relation to rs1837253 genotype merits investigation. Activation of the innate immune system in the epithelium involving rs 1837253 may ultimately influence the development of allergic diseases by modulating TSLP downstream effector pathways. Further investigation into SNP rs1837253-associated epigenetic alterations in airway epithelial cells will also be critical in understanding the mechanisms underlying the involvement of rs1837253 in regulating TSLP secretion.

\section{METHODS}

Study subjects. All studies were approved by Hamilton Integrated Research Ethics Board and subjects provided written informed consent to participate. Eligible subjects were healthy, nonsmoking individuals, 18 to 63 years of age, with no history of use of any nasal, oral, or inhaled corticosteroids within the previous 3 months. All recruited study participants had mouthwash samples collected and skin prick tests performed at visit 1 . Atopy was defined as a positive skin prick test response ( $>2-\mathrm{mm}$ wheal) to at least one of 14 common aeroallergens. In addition, all subjects were confirmed to have no prior history of upper respiratory tract infection within 6 weeks of sample collection. Further subject characteristics are shown in Table 1.

DNA extraction and genotyping. The SNP rs1837253 in the TSLP gene was selected based on previous associations with asthma and related traits as well as the absence of significant linkage disequilibrium with any other SNP. ${ }^{18,20,21}$ DNA was extracted from mouthwash samples using QIAamp DNA Blood Mini Kits (Qiagen, Toronto, ON, Canada) according to the manufacturer's supplementary protocol for mouthwash samples. Genotyping was performed using a commercially available TaqMan genotyping assay for rs1837253 (Assay C_11910823_20, Life Technologies, Burlington, ON, Canada). The genotyping data were verified for Hardy-Weinberg equilibrium by the $\chi^{2}$ test.

Nasal epithelial cell isolation and culture. Primary NECs were derived from the inferior nasal turbinate using Rhino-Probe nasal curettes (Arlington Scientific, Springville, UT). NECs were expanded and cultured as previously described with modification. ${ }^{35}$ Briefly, NECs were maintained in bronchial epithelial growth medium (BEGM; Clonetics, Allendale, NJ) supplemented with SingleQuots (Clonetics). The SingleQuots comprised $2 \mathrm{ml}$ bovine pituitary extract $\left(52 \mu \mathrm{g} \mathrm{ml}^{-1}\right)$ and $0.5 \mathrm{ml}$ each of insulin $\left(5 \mu \mathrm{g} \mathrm{ml}^{-1}\right)$, human epidermal growth factor $\left(0.5 \mathrm{ng} \mathrm{ml}^{-1}\right)$, hydrocortisone $\left(0.5 \mu \mathrm{g} \mathrm{ml}^{-1}\right)$, epinephrine $\left(0.5 \mu \mathrm{g} \mathrm{ml}^{-1}\right)$, transferrin $\left(10 \mu \mathrm{g} \mathrm{ml}^{-1}\right)$, retinoic acid $\left(0.1 \mathrm{ng} \mathrm{ml}^{-1}\right)$, triiodo-L-thyronine $\left(6.5 \mathrm{ng} \mathrm{ml}^{-1}\right)$, and Gentamicin/ Amphotericin-B (GA1000; $50 \mu \mathrm{g} \mathrm{ml}^{-1}$ ). Collected cells were centrifuged and resuspended in BEGM with $10 \% 100 \times$ DNase 1 (Sigma Aldrich, St Louis, MO; at room temperature for $20 \mathrm{~min}$ ). Cells were washed twice and plated on $35 \mathrm{~mm}$ culture dishes (Corning Costar, Corning, NY), coated with Purecol (Inamed, Fremont, CA), and incubated at $37^{\circ} \mathrm{C}, 5 \% \mathrm{CO}_{2}$. Media were changed $24 \mathrm{~h}$ after plating; thereafter, media were replaced every second day until $80-90 \%$ confluence. Subsequently, cells were passaged (P1) using trypsin (Gibco, Burlington, ON, Canada) and further expanded in $75 \mathrm{~cm}^{2}$ flasks (Corning) in BEGM. In this study, second and third passaged cells were used. Viability was assessed by exclusion of Trypan blue dye (Gibco) and the epithelial nature of cells assessed by immunocytochemistry as previously described with modification. ${ }^{51}$ Briefly, selected cultures were fixed in $4 \%$ paraformaldehyde and permeabilized using Triton-X 100 (Sigma). Subsequently, cells were incubated with rabbit anti-cytokeratin 5 antibody (Abcam, Cambridge, UK) overnight at $4{ }^{\circ} \mathrm{C}$. Cells were then exposed to AlexaFluor 488 goat antirabbit IgG antibody (Life Technologies) at room temperature for $1 \mathrm{~h}$ in the dark. Nuclei were stained with DRAQ5 (Abcam) at room temperature for $15 \mathrm{~min}$ and slides were subsequently mounted using Fluoroshield Mounting Medium (Abcam). Negative control slides were prepared by omitting the primary antibody (Figure 1). Fluorescent images were viewed using a Leica SP5 confocal inverted microscope (Leica Microsystems, Concord, ON, Canada) using a $63 \times$ oil immersion objective. Images were captured and viewed using LAS AF (Leica) acquisition software.

Polyl:C stimulation. NEC were seeded onto 24-well plates (Corning) and allowed to grow to $80-90 \%$ confluence. Thereafter, BEGM was replaced with bronchial epithelial basal media (Clonetics) and $1 \%$ fetal bovine serum (R\&D Systems, Minneapolis, MN) before experimental stimulation. NECs were treated with polyI:C (EMD Chemicals, Gibbstown, NJ) as indicated or vehicle control for $24 \mathrm{~h}$ at $37^{\circ} \mathrm{C}$, $5 \% \mathrm{CO}_{2}$.

RNA isolation and reverse transcription. RNA was extracted from a number of different human nasal epithelial samples using the RNeasy Mini-kit (Qiagen, Valencia, CA) according to the manufacturer's instructions. Total RNA in each sample was quantified using a NanoDrop Spectrophotometer (Thermo Fisher, Wilmington, DE). The RNA in each sample time point $(0,3$, and $6 \mathrm{~h})$ from each participant was diluted based on the lowest concentration sample using RNase-free water. The samples were reverse-transcribed using the Quantitect Reverse Transcription kit with genomic DNA wipeout buffer (Qiagen) and complementary DNA were aliquoted and stored at $-80^{\circ} \mathrm{C}$.

Quantitative reverse transcription-PCR. The expression of TSLP was determined by quantitative reverse transcription-PCR using SsoAdvanced Universal SYBR Green Supermix (Bio-Rad, Hercules, CA) with a MX4000 Stratagene detection system according to the manufacturer's instructions. The PrimePCR Human Reference Gene Panel (Bio-Rad) was used to determine glyceraldehyde 3-phosphate dehydrogenase $(G A P D H)$ and tyrosine 3-monooxygenase/tryptophan 5-monooxygenase activation protein, (YWHAZ) to be the optimal reference genes. We used the following primer set for quantitative reverse transcription-PCR: (NM_033035.4 and NM_138551.4), 5'-CT AAGGCTGCCTTAGCTATC- $3^{\prime}$ and $5^{\prime}$-AAGCGACGCCACAATC CTTG- $3^{\prime}$ as previously described. ${ }^{26}$ PrimePCR SYBR green assay for Human GAPDH and YWHAZ were used for reference gene primers (Bio-Rad). All TSLP primer sets (Integrated DNA Technologies, Coralville, IA) were designed and evaluated as per the minimum information for publication of quantitative reverse transcription-PCR experiments (MIQE) guidelines. ${ }^{52}$ Pooled complementary DNA was used to optimize annealing temperature (found to be $60^{\circ} \mathrm{C}$ for all primer sets). The 8-point standard curves were used to verify amplification efficiency for each TSLP isoform primer sets and the GAPDH/YWHAZ reference genes using Sso Advanced SYBR green Supermix (Bio-Rad). Melt curves were used to verify expected single peak amplicons. The threshold cycle $(\mathrm{Ct})$ is inversely correlated with the target mRNA expression level and was defined as the cycle number at which the reporter fluorescence emission exceeded the midpoint along the amplification curve. ${ }^{53}$ The standard $2^{(-\Delta \Delta \mathrm{Ct})}$ formula was used to calculate arbitrary TSLP mRNA concentrations. ${ }^{53}$ The level of TSLP mRNA was normalized to the level of GAPDH/YWHAZ mRNA. 
TSLP measurement. The release of TSLP in cell-free supernatant after $24 \mathrm{~h}$ stimulation with polyI:C was measured using DuoSet enzymelinked immunosorbent assay (ELISA) Development kits (R\&D) as per the manufacturer's instructions.

Statistical analysis. All data are expressed as mean \pm s.e.m. Significance was assumed at $P<0.05$. All analyses were performed with Prism version 5 (GraphPad Software, La Jolla, CA) using nonparametric tests. Differences within groups were assessed by Friedman test with Dunn's post hoc test. Differences between groups (CC and CT vs. TT) were assessed by Mann-Whitney $U$-test.

\section{ACKNOWLEDGMENTS}

We acknowledge Mark Larché (McMaster University) for his valuable scientific input and critical appraisal of the manuscript and Adrian Baatjes (McMaster University) for his assistance with immunofluorescence staining. This work was supported by AllerGen NCE and Canadian Institutes for Health Research MOP 111029 to J.A.D. C.C.K.H. is a recipient of the AllerGen NCE Canadian Allergy and Immune Diseases Advanced Training Initiative (CAIDATI) Award. L.A. is the recipient of the UBC Four-Year Doctoral Fellowship and the AllerGen NCE CAIDATI Award.

\section{DISCLOSURE}

The authors declared no conflict of interest.

c) 2015 Society for Mucosal Immunology

\section{REFERENCES}

1. Holgate, S.T. The epithelium takes centre stage in asthma and atopic dermatitis. Trends Immunol. 28, 248-251 (2007).

2. Kato, A. \& Schleimer, R.P. Beyond inflammation: airway epithelial cells are at the interface of innate and adaptive immunity. Curr. Opin. Immunol. 19, 711-720 (2007).

3. Leonard, W.J. TSLP: finally in the limelight. Nat. Immunol. 3, 605-607 (2002).

4. Soumelis, V. et al. Human epithelial cells trigger dendritic cell mediated allergic inflammation by producing TSLP. Nat. Immunol. 3, 673-680 (2002).

5. Rochman, I., Watanabe, N., Arima, K., Liu, Y.J. \& Leonard, W.J. Cutting edge: direct action of thymic stromal lymphopoietin on activated human CD4 + T cells. J. Immunol. 178, 6720-6724 (2007).

6. Wong, C.K., Hu, S., Cheung, P.F. \& Lam, C.W. Thymic stromal lymphopoietin induces chemotactic and prosurvival effects in eosinophils: implications in allergic inflammation. Am. J. Respir. Cell Mol. Biol. 43, 305315 (2010).

7. Cook, E.B., Stahl, J.L., Schwantes, E.A., Fox, K.E. \& Mathur, S.K. IL-3 and TNFalpha increase thymic stromal lymphopoietin receptor (TSLPR) expression on eosinophils and enhance TSLP-stimulated degranulation. Clin. Mol. Allergy 10, 8 (2012).

8. Siracusa, M.C. et al. TSLP promotes interleukin-3-independent basophil haematopoiesis and type 2 inflammation. Nature 477, 229-233 (2011).

9. Allakhverdi, Z. et al. CD34+ hemopoietic progenitor cells are potent effectors of allergic inflammation. J. Allergy Clin. Immunol. 123, 472-478 (2009).

10. Hui, C.C., Rusta-Sallehy, S., Asher, I., Heroux, D. \& Denburg, D.A. The effects of thymic stromal lymphopoietin and IL-3 on human eosinophilbasophil lineage commitment: relevance to atopic sensitization. Immun. Inflamm. Dis. 2, 44-55 (2014).

11. Ying, S. et al. Thymic stromal lymphopoietin expression is increased in asthmatic airways and correlates with expression of Th2-attracting chemokines and disease severity. J. Immunol. 174, 8183-8190 (2005).

12. Zhou, B. et al. Thymic stromal lymphopoietin as a key initiator of allergic airway inflammation in mice. Nat. Immunol. 6, 1047-1053 (2005).

13. Gauvreau, G.M. et al. Effects of an anti-TSLP antibody on allergen-induced asthmatic responses. N. Engl. J. Med. 370, 2102-2110 (2014).

14. Hirota, T. et al. Genome-wide association study identifies three new susceptibility loci for adult asthma in the Japanese population. Nat. Genet. 43, 893-896 (2011).
15. Torgerson, D.G. et al. Meta-analysis of genome-wide association studies of asthma in ethnically diverse North American populations. Nat. Genet. 43, 887-892 (2011).

16. Ferreira, M.A. et al. Genome-wide association analysis identifies 11 risk variants associated with the asthma with hay fever phenotype. J. Allergy Clin. Immunol. 133, 1564-1571 (2014).

17. Gudbjartsson, D.F. et al. Sequence variants affecting eosinophil numbers associate with asthma and myocardial infarction. Nat. Genet. 41, 342-347 (2009).

18. He, J.Q. et al. A thymic stromal lymphopoietin gene variant is associated with asthma and airway hyperresponsiveness. J. Allergy Clin. Immunol. 124, 222-229 (2009).

19. Moffatt, M.F. et al. A large-scale, consortium-based genomewide association study of asthma. N. Engl. J. Med. 363, 1211-1221 (2010).

20. Hunninghake, G.M. et al. TSLP polymorphisms are associated with asthma in a sex-specific fashion. Allergy 65, 1566-1575 (2010).

21. Bunyavanich, S. et al. Thymic stromal lymphopoietin (TSLP) is associated with allergic rhinitis in children with asthma. Clin. Mol. Allergy 9, 1 (2011).

22. Allakhverdi, Z. et al. Thymic stromal lymphopoietin is released by human epithelial cells in response to microbes, trauma, or inflammation and potently activates mast cells. J. Exp. Med. 204, 253-258 (2007).

23. Kato, A., Favoreto, S. Jr, Avila, P.C. \& Schleimer, R.P. TLR3- and Th2 cytokine-dependent production of thymic stromal lymphopoietin in human airway epithelial cells. J. Immunol. 179, 1080-1087 (2007).

24. Lee, H.C. \& Ziegler, S.F. Inducible expression of the proallergic cytokine thymic stromal lymphopoietin in airway epithelial cells is controlled by NFkappaB. Proc. Natl. Acad. Sci. USA 104, 914-919 (2007).

25. Hunninghake, G.M. et al. Sex-stratified linkage analysis identifies a femalespecific locus for IgE to cockroach in Costa Ricans. Am. J. Respir. Crit. Care Med. 177, 830-836 (2008).

26. Harada, M. et al. Functional analysis of the thymic stromal lymphopoietin variants in human bronchial epithelial cells. Am. J. Respir. Cell Mol. Biol. 40, 368-374 (2009).

27. Harada, M. et al. Thymic stromal lymphopoietin gene promoter polymorphisms are associated with susceptibility to bronchial asthma. Am. J. Respir. Cell Mol. Biol. 44, 787-793 (2011).

28. Zhang, Y., Song, X., Zhao, Y., Zhang, L. \& Bachert, C. Single nucleotide polymorphisms in thymic stromal lymphopoietin gene are not associated with allergic rhinitis susceptibility in Chinese subjects. BMC Med. Genet. 13, 79 (2012)

29. Xu, G. et al. Opposing roles of IL-17a and IL-25 in the regulation of TSLP production in human nasal epithelial cells. Allergy 65, 581-589 (2010).

30. Zhu, D.D., Zhu, X.W., Jiang, X.D. \& Dong, Z. Thymic stromal lymphopoietin expression is increased in nasal epithelial cells of patients with mugwort pollen sensitive-seasonal allergic rhinitis. Chin. Med. J. (Engl.) 122, 2303-2307 (2009).

31. Mou, Z. et al. Overexpression of thymic stromal lymphopoietin in allergic rhinitis. Acta Otolaryngol. 129, 297-301 (2009).

32. Kimura, S. et al. Increased expression and role of thymic stromal lymphopoietin in nasal polyposis. Allergy Asthma Immunol. Res. 3, 186-193 (2011).

33. Yeh, C.Y., Yeh, T.H., Jung, C.J., Chen, P.L., Lien, H.T. \& Chia, J.S. Activated human nasal epithelial cells modulate specific antibody response against bacterial or viral antigens. PLoS One 8, e55472 (2013).

34. Uller, L. et al. Double-stranded RNA induces disproportionate expression of thymic stromal Iymphopoietin versus interferon-beta in bronchial epithelial cells from donors with asthma. Thorax 65, 626-632 (2010).

35. Hui, C.C. et al. Tcell-mediated induction of thymic stromal lymphopoietin in differentiated human primary bronchial epithelial cells. Clin. Exp. Allergy 44, 953-964 (2014).

36. Lee, H.C. et al. Thymic stromal lymphopoietin is induced by respiratory syncytial virus-infected airway epithelial cells and promotes a type 2 response to infection. J. Allergy Clin. Immunol. 130, 1187-1196 e1185 (2012).

37. Miazgowicz, M.M., Elliott, M.S., Debley, J.S. \& Ziegler, S.F. Respiratory syncytial virus induces functional thymic stromal lymphopoietin receptor in airway epithelial cells. J. Inflamm. Res. 6, 53-61 (2013).

38. Demoly, P. et al. C-fos proto-oncogene expression in bronchial biopsies of asthmatics. Am. J. Respir. Cell Mol. Biol. 7, 128-133 (1992). 
39. Conde, L. et al. Pupasuite: finding functional single nucleotide polymorphisms for large-scale genotyping purposes. Nucleic Acids Res. 34, W621-W625 (2006).

40. Shikotra, A. et al. Increased expression of immunoreactive thymic stromal lymphopoietin in patients with severe asthma. J. Allergy Clin. Immunol. 129, 104-111, e101-109 (2012).

41. Gao, P.S. et al. Genetic variants in thymic stromal lymphopoietin are associated with atopic dermatitis and eczema herpeticum. J. Allergy Clin. Immunol. 125, 1403-1407, e1404 (2010).

42. O'Byrne, P.M., Pedersen, S., Lamm, C.J., Tan, W.C. \& Busse, W.W. Severe exacerbations and decline in lung function in asthma. Am. J. Respir. Crit. Care Med. 179, 19-24 (2009).

43. Hewson, C.A., Jardine, A., Edwards, M.R., Laza-Stanca, V. \& Johnston, S.L. Toll-like receptor 3 is induced by and mediates antiviral activity against rhinovirus infection of human bronchial epithelial cells. J. Virol. 79 , 12273-12279 (2005).

44. Hershenson, M.B. \& Johnston, S.L. Rhinovirus infections: more than a common cold. Am. J. Respir. Crit. Care Med. 174, 1284-1285 (2006).

45. Luo, Y., Zhou, B., Zhao, M., Tang, J. \& Lu, Q. Promoter demethylation contributes to TSLP overexpression in skin lesions of patients with atopic dermatitis. Clin. Exp. Dermatol. 39, 48-53 (2014).
46. Wang, I.J., Chen, S.L., Lu, T.P., Chuang, E.Y. \& Chen, P.C. Prenatal smoke exposure, DNA methylation, and childhood atopic dermatitis. Clin. Exp. Allergy 43, 535-543 (2013).

47. Lambrecht, B.N. \& Hammad, H. The airway epithelium in asthma. Nat. Med. 18, 684-692 (2012).

48. Vercelli, D. Genetics, epigenetics, and the environment: switching, buffering, releasing. J. Allergy Clin. Immunol. 113, 381-386, quiz 387 (2004).

49. Suzuki, M.M. \& Bird, A. DNA methylation landscapes: provocative insights from epigenomics. Nat. Rev. Genet. 9, 465-476 (2008).

50. Poetsch, A.R. \& Plass, C. Transcriptional regulation by DNA methylation. Cancer Treat. Rev. 37 (Suppl 1), S8-S12 (2011).

51. Wark, P.A. et al. Asthmatic bronchial epithelial cells have a deficient innate immune response to infection with rhinovirus. J. Exp. Med. 201, 937-947 (2005).

52. Bustin, S.A. et al. The MIQE guidelines: minimum information for publication of quantitative real-time PCR experiments. Clin. Chem. 55, 611-622 (2009).

53. Livak, K.J. \& Schmittgen, T.D. Analysis of relative gene expression data using real-time quantitative PCR and the 2(-Delta Delta C(T)) Method. Methods 25, 402-408 (2001). 